\title{
ÍNDICE DE DESARROLLO HUMANO: UNA MIRADA DESDE ECUADOR
}

\author{
HUMAN DEVELOPMENT INDEX: \\ A LOOK FROM ECUADOR
}

ESTEFANÍA SUÁREZ ${ }^{1}$

Recibido: 12 de abril

Aceptado: 1 de mayo

\footnotetext{
${ }^{1}$ Economista por la Pontifica Universidad Católica del Ecuador, licenciada en Derecho, Economía y Administración con mención de Economía y Administración por la Universidad Grenoble Alpes (Francia). Actualmente trabaja en Grupo Faro. estefania.s.suarez@gmail.com. +5939925222566
} 



\title{
ÍNDICE DE DESARROLLO HUMANO: UNA MIRADA DESDE ECUADOR
}

\section{HUMAN DEVELOPMENT INDEX: A LOOK FROM ECUADOR}

Estefanía Suárez

\begin{abstract}
Palabras clave: índice de desarrollo humano, índice de desarrollo humano no extractivista, valor agregado bruto no extractivista
\end{abstract}

Keywords: human development index, non-extractivist human development index, non-extractivist gross added value

\section{RESUMEN}

El índice de desarrollo humano revolucionó la forma de cuantificar el desarrollo de un país al incluir indicadores adicionales a aquellos relativos a los ingresos. Si bien este índice es útil para comparar el desarrollo de distintos países, puede no ser adecuado para evidenciar el desarrollo en un país en particular dado que omite aspectos relevantes sobre el contexto de cada país. Por ello, es necesario revisarlo y ajustarlo para que logre reflejar de mejor mane- ra el desarrollo de cada país. Se observó que el índice de desarrollo humano calculado a nivel nacional para Ecuador esconde las disparidades existentes entre las provincias del país. Adicionalmente, se encontró que el índice de desarrollo humano de algunas provincias se distorsiona debido a las altas cifras registradas en la dimensión de estándar de vida; en consecuencia, un mejor indicador del desarrollo humano a nivel provincial para Ecuador es el índice de 
desarrollo humano no extractivista, el cual excluye el componente de activi- dades extractivistas del valor agregado bruto provincial.

\section{ABSTRACT}

The human development index revolutionized the way in which the development of a country is quantified, by including additional indicators to those referring to income. Although this index is useful to compare the development of different countries, it may not be adequate to reflect a country's development, given that it omits relevant aspects regarding the context of each country. Therefore, it is necessary to review and adjust the human development index, in order to improve the information regarding the development of each country. It was observed that when calculating the human development index, at national level, for Ecuador, the index leaves out the existing disparities between the provinces of the country. Additionally, it was found that the human development index of some provinces is distorted due to high figures registered in the standard of living dimension; consequently, a better indicator of human development at the provincial level for Ecuador is the non-extractivist index of human development, which excludes the extractivist activities component of the provincial gross added value.

\section{INTRODUCCIÓN}

El desarrollo humano refleja los avances en bienestar, libertad o felicidad de las personas, según el enfoque bajo el cual se esté realizando el análisis. No obstante, resulta complejo entender cuáles son en realidad los factores que elevan el bienestar o calidad de vida de las personas y cuáles son aquellos que en lugar de elevarla lo disminuyen. Es por esta razón que medir el bienestar que las personas poseen es sumamente difícil.

Hoy en día, una medida ampliamente utilizada a nivel mundial para medir qué tan elevado es el desarrollo humano de los países, es el Índice de Desarrollo Humano (IDH) del Programa de las Naciones Unidas para el Desarrollo (PNUD). Este índice surgió de la crítica de 
que el desarrollo de los países no puede ser medido solamente a través de los ingresos nacionales, sino que hay muchos otros factores que deben ser tomados en cuenta. El índice que fue una revolución para su época incorporó, además del ingreso, la esperanza de vida y la alfabetización (PNUD, 2010).

A pesar de que en 1990 se consideraba al IDH como una evolución en la forma de entender y medir el desarrollo de las personas, el índice ha recibido críticas a lo largo de los años. Una parte de las críticas hace referencia al hecho de que el desarrollo humano es una noción muy amplia y que el IDH es limitado y reduccionista dado que ignora aspectos relevantes para la vida de las personas (Ranis, G., Stewart F. \& Samman, E., 2006). Otros autores critican este índice porque al utilizarlo para cuantificar el desarrollo de varios países, se asume que el bienestar de las personas depende de los mismos factores y que todos valoran estos factores por igual. No obstante, algunas investigaciones demuestran que los aspectos que afectan al bienestar de las personas son distintos para diferentes países (Kroll, 2015).

EI PNUD ha incorporado otros índices, como el índice de desarrollo humano ajustado por desigualdad y el índice de igualdad de género, para fortalecer el enfoque inicial de desarrollo humano, además ha realizado mejoras metodológicas para robustecer el índice de desarrollo humano tradicional (PNUD, 2010). Asimismo, ha afirmado que lo que busca el índice tradicional, no es ser un indicador insuperable de bienestar sino direccionar el debate a que el desarrollo y las métricas que se utilicen para medirlo, se centren en el ser humano. En consecuencia, menciona que se puede adaptar el IDH a la realidad y necesidades locales de los países para que las mediciones reflejen de manera más adecuada los problemas para el desarrollo de cada país (PNUD, 2015).

En este sentido, el objetivo de este estudio es extender el IDH de Ecuador a sus provincias para observar qué tan adecuada sería esta medición a un nivel más desagregado para el caso ecuatoriano. Para ello; se replica el índice a nivel provincial, se analizan los resultados y en función de estos, se realizan ajustes al índice para que este pueda reflejar de mejor manera el desarrollo humano de las provincias del país. 


\section{REVISIÓN DE LA LITERATURA}

\section{Fundamento teórico y filosófico}

El desarrollo de las sociedades y de sus individuos ha sido discutido desde diferentes áreas del conocimiento como la economía, sociología, psicología y filosofía. Particularmente, desde la ciencia económica, el desarrollo era entendido como un fenómeno puramente económico en el sentido de que el desarrollo de un país implicaba que este alcance una tasa de crecimiento sostenida del ingreso per cápita en el tiempo. Por tanto, el desarrollo se cuantificaba en relación con cuántos bienes y servicios una persona promedio podía consumir (Todaro \& Smith, 2015).

Sin embargo, en las décadas siguientes a la Segunda Guerra Mundial, muchos países habían alcanzado sus objetivos de crecimiento, pero la calidad de vida de sus habitantes no necesariamente había cambiado. En ese momento, era claro que la concepción de desarrollo debía repensarse. Así, desde la década de 1970, aunque el incremento del ingreso no dejó de ser un eje fundamental, se redefinió al desarrollo como un proceso de reducción de la pobreza, desigualdad y desempleo. Se estableció entonces que el desarrollo debía ser considerado "como un proceso multidimensional, que implica cambios importantes en las estructuras sociales, las actitudes popu- lares y en las instituciones nacionales, así como la aceleración del crecimiento económico, la reducción de la desigualdad y la erradicación de la pobreza" (Todaro \& Smith, 2015: 18).

En 1990, se presentó el concepto de desarrollo humano en el primer Informe de Desarrollo Humano del PNUD. Este marcó el inicio de una nueva concepción, así como de una evolución de lo que se consideraba como desarrollo (Sagar \& Najam, 1999). Esta nueva concepción, partió de la crítica de que el desarrollo no puede ser entendido ni medido únicamente a través del ingreso, sino que se deben incluir otras variables como salud y educación (PNUD, 2010). Este enfoque de desarrollo humano, se fundamentó filosóficamente en el enfoque de capacidades propuesto por Amartya Sen.

El enfoque de capacidades entiende a la vida de las personas como un conjunto de capacidades para ser y hacer al cual Ilama funcionamientos. En este contexto, las capacidades reflejan distintas combinaciones de funcionamientos que las personas pueden alcanzar, en otras palabras, la capacidad es un reflejo de la libertad que las personas poseen para elegir entre diferentes formas de vida. En este sentido, se considera que la vida se compone por una combinación 
de varios funcionamientos por lo cual no es correcto medir el desarrollo de las personas solamente a través del ingreso. Según este enfoque, no solo se debe considerar los funcionamientos sino también la capacidad de las personas de alcanzarlos (Sen, 2012).

Sen manifiesta que existen algunos funcionamientos y los clasifica en elementales y complejos. Los elementales hacen referencia a cuestiones como estar en la capacidad de evitar la muerte y de poder nutrirse adecuadamente, y los complejos se relacionan con aspectos como tener respeto hacia uno mismo y participar en la vida comunitaria (Sen, 2012). No obstante, no llega a describir explícitamente cuáles son todos los funcionamientos a los que se refiriere. En este respecto, Martha Nussbaum desarrolla en su artículo "Capabilities as Fundamental Entitlements: Sen and Social Justice" una lista de diez capacidades que considera son fundamentales para que las personas puedan disfrutar de una vida digna (Nussbaum, 2003). En este mismo documento, Nussbaum define a las capacidades como "lo que las personas en realidad pueden hacer y ser (Nussbaum, 2003: 39) y mantiene que la lista de capacidades no es una lista definitiva, sino que puede ser sujeta de revisión y que puede hacerse más específica de acuerdo con cada sociedad y sus culturas.

Adicionalmente, otro enfoque filosófico de Amartya Sen que también se encuentra estrechamente relacionado al desarrollo humano es el de desarrollo como expansión de libertades. Según este enfoque, el desarrollo humano puede ser entendido como "un proceso de expandir las libertades reales que las personas disfrutan" (Sen, 1999: 3). Este enfoque, aunque es más amplio que los enfoques basados en crecimiento económico, no deja de considerar al ingreso como una variable relevante para lograr expandir las libertades de los individuos, sin embargo, considera que este es solo un medio para alcanzar el desarrollo y no el fin. Este enfoque reconoce que las libertades dependen tanto en factores económicos como en derechos políticos y civiles, y que para alcanzar el desarrollo es necesario retirar las barreras que privan a los individuos de sus libertades. Se identifican cinco tipos de libertades: políticas, facilidades económicas, oportunidades sociales, garantías de transparencia, seguridad; estas se pueden ver afectadas por factores como la salud y la educación de los individuos (Sen, 1999). 


\section{ESTUDIOS EMPÍRICOS}

Se han realizado varios esfuerzos por innovar y adaptar el IDH para reflejar de mejor manera la realidad local de países o regiones en particular. En América Latina, Argentina, Colombia y Costa Rica han realizado ajustes al IDH en diferentes aspectos. En Argentina se construyó un IDH ampliado, con el cual se aumentaron indicadores a todas las dimensiones del IDH tradicional. Al calcular este índice a nivel provincial se pudo observar que las disparidades entre las provincias eran significativamente más pronunciadas que las que reflejaba el IDH tradicional (PNUD, 2009). Colombia realizó un IDH corregido por violencia, el cual añade una dimensión y un indicador al índice convencional. La dimensión adicional es una referente a violencia, la cual se mide a través de la tasa de homicidios por cada 100.000 habitantes. Según este índice Colombia perdía 14 escalones en el ranking mundial de los países según su nivel de desarrollo humano del año 2002
(PNUD, 2003). En Costa Rica, se creó un IDH cantonal el cual buscaba reflejar la disminución en la calidad de vida que causa la inseguridad ciudadana. Para ello, realizaron un IDH cantonal ajustado por niveles de seguridad, que incluía variables de victimización por homicidio de diversa índole, los resultados mostraron nuevamente que los cantones se ubicaban en posiciones muy distintas en relación con el ranking original (PNUD, 2006).

A parte de estos ensayos se han hecho muchos otros esfuerzos alrededor del mundo para mejorar las mediciones del desarrollo humano, adaptando o corrigiendo los índices por diferentes aspectos como cuestiones medio ambientales, desigualdad de género, entre otras, o calculando los índices a niveles más desagregados. Estos ejercicios han vislumbrado las diferencias en el desarrollo humano entre regiones de los países o grupos poblacionales dentro de ellos (PNUD, 2015). 


\section{METODOLOGÍA}

Para calcular el IDH a nivel provincial para Ecuador, se utilizó la metodología propuesta por el PNUD más actualizada hasta la fecha. Según esta, el IDH contempla tres dimensiones y cuatro indicadores, los cuales se pueden observar en la figura.

Dimensiones e indicadores del IDH

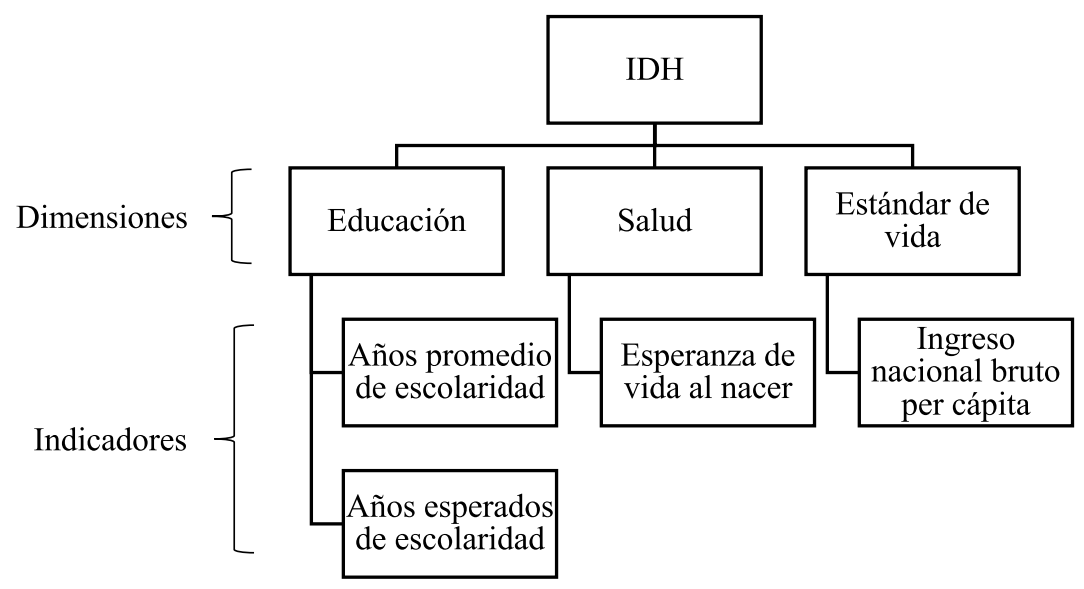

Fuente: PNUD, 2016a

Estos indicadores se definen, según el Informe de Desarrollo Humano de 2016 (PNUD, 2016a), de la siguiente manera:

Años promedio de escolaridad: número promedio de años de educación recibidos por las personas de 25 años o más. El número de años se calcula en base a los niveles de logros educativos y su duración oficial.
Años esperados de escolaridad: número de años de escolaridad, que un niño que se encuentra en edad de comenzar la escuela puede esperar recibir en el caso de que las tasas de matriculación por edad se mantengan a lo largo de su vida.

Esperanza de vida al nacer: número de años que se espera que viva un recién nacido si los patrones de las 
tasas de mortalidad por edad vigentes en el momento de su nacimiento se mantienen a lo largo de su vida.

\section{Ingreso nacional bruto estima-} do per cápita: ingresos totales generados por una economía menos los pagos al resto del mundo por el uso de factores de producción que son de su propiedad, ajustado por la paridad de poder de compra y dividido para la población a mitad del año.

\section{Construcción del índice}

Según la nota técnica del Informe de Desarrollo Humano del año 2016 (PNUD, 2016b), el IDH se calcula en dos pasos:

Paso 1: Crear un índice para cada dimensión

Se estandarizan los indicadores de cada dimensión, transformándolos a un índice que tenga una escala de $0 \mathrm{a}$ 1. Para ello, se utilizan valores mínimos y máximos los cuales se presentan en la tabla 1.

Tabla 1: Mínimos y máximos de cada indicador del IDH

\begin{tabular}{|l|l|c|c|}
\hline \multicolumn{1}{|c|}{ Dimensión } & \multicolumn{1}{c|}{ Indicador } & Mínimo & Máximo \\
\hline Salud & Esperanza de vida (años) & 20 & 85 \\
\hline Educación & Años esperados de escolaridad & 0 & 18 \\
\cline { 2 - 4 } & Años promedio de escolaridad & 0 & 15 \\
\hline Estándar de vida & Ingreso nacional bruto per cápita (\$) & 100 & 75.000 \\
\hline
\end{tabular}

Fuente: PNUD, 2016b

Según el PNUD (2015), estos valores mínimos y máximos se han planteado en forma de ceros naturales, metas aspiracionales, o se han fundamentado en estudios de diversos autores. Por un lado, los valores máximos de los indicadores esperanza de vida, años esperados de escolaridad y años promedio de escolaridad, se han planteado como valores deseables y en ese sentido representan metas aspiracionales. Por otro lado, los valores 0 en años esperados de escola- ridad y años promedio de escolaridad representan ceros naturales ya que es posible que existan sociedades sin ningún tipo de educación formal. En relación con el indicador de la salud, el valor mínimo que puede tomar la esperanza de vida es 20 años según un estudio realizado que demuestra que, de ser menor, la sociedad no podría reproducirse. Por otra parte, el valor mínimo de estándar de vida (\$100) busca reflejar la economía de subsistencia que no se cuantifica en 
las estadísticas oficiales y el valor máximo (\$75.000) se fundamenta en que un aumento de los ingresos, más allá de este monto, no conllevaría a un incremento en el bienestar de la persona.

En función de estos límites mínimos y máximos se calcula un índice para cada dimensión, como se muestra a continuación:

$$
\text { Índice }=\frac{\text { valor actual }- \text { mínimo }}{\text { máximo }- \text { mínimo }}
$$

En este paso, se deben tomar en consideración dos aspectos relevantes: i) el índice de educación está compuesto por dos subíndices (uno para cada indicador), los cuales se agregan mediante un promedio simple; ii) para calcular el índice de estándar de vida, se utiliza el logaritmo natural del indicador, con el objetivo de capturar el efecto decreciente que el ingreso tiene en la expansión de las capacidades (PNUD, 2016b).

Paso 2: Agregar las dimensiones

Para agregar las dimensiones se utiliza la media geométrica, como se muestra a continuación:

$$
\text { IDH=(Índice } \left.\text { Salud }_{1} \times \text { Índice }_{\text {Educación }} \times \text { Índice }_{\text {Ingress }}\right)^{1 / 3}
$$

A pesar de que, en la primera versión del IDH, se utilizaba un promedio simple, desde el año 2010, se utiliza la media geométrica por dos razones. La primera razón es que la media geométrica penaliza la desigualdad entre las dimensiones, es decir, si los logros alcanzados en cada una de las dimensiones difieren en gran medida, el IDH resultante será menor. Segundo, si se utiliza la media simple o aritmética, se asume que las dimensiones son sustitutos perfectos entre sí; sin embargo, un incremento en una dimensión no debería compensar una disminución en otra (PNUD, 2015).

Una vez calculado el IDH, se puede clasificar a los países como países con desarrollo humano muy alto, alto, medio o bajo. Según el PNUD, los países que tienen desarrollo humano muy alto, poseen un IDH de 0,8 o mayor, si su IDH se encuentra en el rango de 0,7-0,799 tienen un desarrollo alto, si su IDH es 0,5500,699 tienen un desarrollo medio y si es menor a 0,550, tienen un desarrollo humano bajo (PNUD, 2016b).

\section{Datos y fuentes de información}

Para calcular los índices a nivel provincial se utilizaron las siguientes variables:

Esperanza de vida: promedio de años que las personas viven en cada provincia.

VAB provincial per cápita: valor agregado bruto anual de una provincia, dividido para la población de la provincia respectiva. 
Promedio de años de escolaridad: número promedio de años escolares aprobados por la población de 24 años y más en el sistema educativo formal en los siguientes niveles de educación: general básica, bachillerato, educación superior universitaria o técnica y postgrado.
Esperanza de vida escolar: promedio de años de educación que una persona de cinco años espera recibir si permanece en el sistema educativo.

Las fuentes de información que se utilizaron son bases de datos oficiales provenientes del Instituto Nacional de Estadística y Censos (INEC) y del Banco Central del Ecuador. 


\section{RESULTADOS}

En la tabla 2 se muestran los in- índice de desarrollo humano a nivel prodicadores con su respectivo índice y el vincial.

Tabla 2: Índice de desarrollo humano a nivel provincial

\begin{tabular}{|c|c|c|c|c|c|c|c|c|}
\hline Provincia & $\begin{array}{c}\text { Esperanza } \\
\text { de vida } \\
\text { al nacer } \\
(\text { años) }\end{array}$ & $\begin{array}{l}\text { Años pro- } \\
\text { medio de } \\
\text { escolaridad } \\
\text { (años) }^{b}\end{array}$ & $\begin{array}{l}\text { Años espe- } \\
\text { rados de } \\
\text { escolaridad } \\
\text { (años) }\end{array}$ & $\begin{array}{l}\text { VAB per } \\
\text { cápita }(\$)^{d}\end{array}$ & $\begin{array}{l}\text { Índice de } \\
\text { esperanza } \\
\text { de vida }^{\mathrm{e}}\end{array}$ & $\begin{array}{l}\text { Índice de } \\
\text { educación }\end{array}$ & $\begin{array}{l}\text { Índice } \\
\text { estándar } \\
\text { de vida }^{\mathrm{e}}\end{array}$ & $\begin{array}{c}\text { Índice de } \\
\text { Desarrollo } \\
\text { Humano } \\
\left(\mathrm{IDH}^{\mathrm{e}}\right)\end{array}$ \\
\hline Azuay & 78,6 & 10 & 14,8 & 4717,6 & 0,902 & 0,745 & 0,582 & 0,731 \\
\hline Bolívar & 76,4 & 9,1 & 14,5 & 2408,8 & 0,868 & 0,707 & 0,481 & 0,665 \\
\hline Cañar & 77,3 & 8,5 & 13,3 & 3135,1 & 0,882 & 0,653 & 0,520 & 0,669 \\
\hline Carchi & 77 & 9,2 & 14,3 & 2403,8 & 0,877 & 0,704 & 0,480 & 0,667 \\
\hline Chimborazo & 75,6 & 8,7 & 14,3 & 4491,6 & 0,855 & 0,687 & 0,575 & 0,696 \\
\hline Cotopaxi & 76,1 & 8,9 & 14,1 & 2404,3 & 0,863 & 0,689 & 0,480 & 0,659 \\
\hline El Oro & 76,2 & 10,3 & 14,0 & 4793,6 & 0,865 & 0,733 & 0,585 & 0,718 \\
\hline Esmeraldas & 74,3 & 10 & 13,8 & 3273,3 & 0,835 & 0,717 & 0,527 & 0,681 \\
\hline Galápagos & 77 & 12,4 & 13,5 & 6566,0 & 0,877 & 0,787 & 0,632 & 0,758 \\
\hline Guayas & 75 & 10,7 & 13,9 & 6426,7 & 0,846 & 0,742 & 0,629 & 0,734 \\
\hline Imbabura & 77 & 9,7 & 14,6 & 3697,5 & 0,877 & 0,729 & 0,545 & 0,704 \\
\hline Loja & 78,7 & 10,5 & 14,2 & 4292,9 & 0,903 & 0,745 & 0,568 & 0,726 \\
\hline Los Ríos & 73,6 & 9 & 13,5 & 3600,1 & 0,825 & 0,674 & 0,541 & 0,670 \\
\hline Manabí & 75,8 & 9,3 & 14,1 & 4093,2 & 0,858 & 0,700 & 0,561 & 0,696 \\
\hline Morona Santiago & 75,4 & 10,3 & 13,8 & 2277,5 & 0,852 & 0,727 & 0,472 & 0,664 \\
\hline Nacional & 76,7 & 10,2 & 14,2 & 5416,5 & 0,872 & 0,734 & 0,603 & 0,728 \\
\hline Napo & 75,6 & 10 & 13,6 & 3053,5 & 0,855 & 0,711 & 0,516 & 0,680 \\
\hline Orellana & 75,6 & 9,5 & 13,8 & 17206,9 & 0,855 & 0,700 & 0,778 & 0,775 \\
\hline Pastaza & 75,6 & 10,3 & 14,3 & 3851,2 & 0,855 & 0,741 & 0,552 & 0,704 \\
\hline Pichincha & 77,8 & 11,6 & 15,1 & 9599,8 & 0,889 & 0,807 & 0,689 & 0,791 \\
\hline Santa Elena & 77,9 & 9,5 & 13,7 & 2371,0 & 0,891 & 0,697 & 0,478 & 0,667 \\
\hline Santo Domingo & 75,6 & 9,9 & 13,5 & 3961,8 & 0,855 & 0,704 & 0,556 & 0,694 \\
\hline Sucumbíos & 75,6 & 10 & 13,9 & 8608,8 & 0,855 & 0,720 & 0,673 & 0,746 \\
\hline Tungurahua & 77,2 & 10 & 14,9 & 4124,8 & 0,880 & 0,747 & 0,562 & 0,718 \\
\hline Zamora Chinchipe & 75,6 & 9,9 & 13,5 & 2971,9 & 0,855 & 0,704 & 0,512 & 0,676 \\
\hline
\end{tabular}

Fuente: a) Compendio Estadístico del año 2016 del INEC, b) ENEMDU 2017 (INEC) en SICES, c) Variable construida a partir de la sintaxis proporcionada SICES, utilizando los datos de ENEMDU de diciembre de 2017 d) Cuentas Regionales del BCE 2016, población de la ENEMDU de diciembre 2017, e) cálculos propios 
Los resultados obtenidos muestran que la provincia con mayor desarrollo humano es Pichincha seguida de Orellana, Galápagos y Sucumbíos, mientras que las menos desarrolladas son Bolívar, Morona Santiago y Cotopaxi. En la tabla 3, las provincias se encuentran ordenadas de manera descendente en función de su desarrollo humano y se categoriza a las provincias según su nivel de desarrollo humano, en función de los rangos propuestos por el PNUD. Según dicha clasificación, todas las provincias tienen un desarrollo humano alto o medio, y en general Ecuador, presenta un desarrollo humano alto.

Tabla 3: Categorías de desarrollo humano

\begin{tabular}{|c|c|c|}
\hline & Provincia & IDH \\
\hline 1 & Pichincha & 0,791 \\
\hline 2 & Orellana & 0,775 \\
\hline 3 & Galápagos & 0,758 \\
\hline 4 & Sucumbíos & 0,746 \\
\hline 5 & Guayas & 0,734 \\
\hline 6 & Azuay & 0,731 \\
\hline 7 & Nacional & 0,728 \\
\hline 8 & Loja & 0,726 \\
\hline 9 & El Oro & 0,718 \\
\hline 10 & Tungurahua & 0,718 \\
\hline 11 & Pastaza & 0,704 \\
\hline 12 & Imbabura & 0,704 \\
\hline 13 & Chimborazo & 0,696 \\
\hline 14 & Manabí & 0,696 \\
\hline 15 & Santo Domingo & 0,694 \\
\hline 16 & Esmeraldas & 0,681 \\
\hline 17 & Napo & 0,680 \\
\hline 18 & Zamora Chinchipe & 0,676 \\
\hline 19 & Los Ríos & 0,670 \\
\hline 20 & Cañar & 0,669 \\
\hline 21 & Santa Elena & 0,667 \\
\hline 22 & Carchi & 0,667 \\
\hline 23 & Bolívar & 0,665 \\
\hline 24 & Morona Santiago & 0,664 \\
\hline 25 & Cotopaxi & 0,659 \\
\hline
\end{tabular}$\quad$ Desarrollo Humano Alto

Fuente: cálculos propios 
Como se puede observar en el gráfico 1, no existen provincias que presenten un IDH demasiado alejado de la media, pues no existe ningún valor atípico. Sin embargo, se puede apreciar que existe una dispersión mayor hacia el valor máximo del diagrama de caja y bigotes. Lo que implica que existe una mayor dispersión entre las provincias de alto desarrollo humano en relación con las de desarrollo humano medio.

\section{Gráfico 1: Caja y bigotes del IDH}

IDH

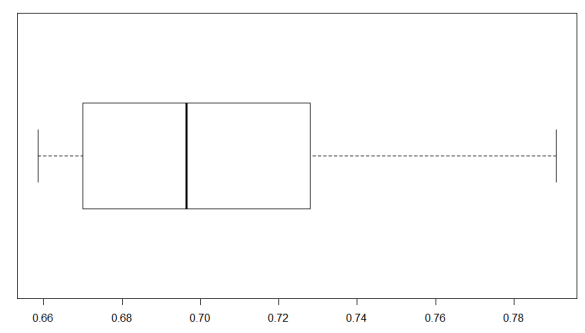

Fuente: cálculos propios

Sin embargo, cabe realizar un análisis de por qué Orellana y Sucumbíos se muestran como unas de las provincias más desarrolladas de Ecuador. Este resultado parecería contradictorio con sus estadísticas de educación y salud, en particular con los años promedio de educación y la esperanza de vida, las cuales son bajas en relación con las otras dos provincias más desarrolladas, Pichincha y Galápagos. Asimismo, se muestra contrario a los resultados obtenidos en otros estudios, por ejemplo, según Burgos y Cando (2016) Orellana y Sucumbíos presentan un índice de pobreza multidimensional de alrededor de 0,2 mientras que Pichincha y Galápagos presenta un índice de pobreza multidimensional menor a 0,09.

Al observar el VAB per cápita de estas provincias (tabla 2) se puede apreciar que exhiben un VAB per cápita considerablemente más alto comparado al resto de provincias. El VAB per cápita de Orellana es $\$ 17.206,9$ y el de Sucumbíos $\$ 8.608,8$, mientras que a nivel nacional el VAB per cápita es solo $\$ 5.416,5$. Estas altas cifras registradas en el VAB per cápita aparecen como consecuencia de la extracción minera y petrolera. Pues, según las Cuentas Regionales del Banco Central del Ecuador, en el año 2016, el 84,2\% del VAB de Orellana correspondía a las actividades de "explotación de minas y canteras" la cual incluye también la explotación petrolera, y en Sucumbíos, el 51,7 \% del VAB se debe a la misma actividad.

Debido a los altos niveles de VAB per cápita que presentan estas provincias, se considera que su IDH podría estar distorsionado. Por esta razón, se recalculó el IDH a nivel provincial excluyendo el componente de explotación de minas y canteras del VAB per cápita. Los resultados de este nuevo índice, al que se llamó IDH no extractivista, se muestran en tabla 4. 
Tabla 4: Índice de desarrollo humano no extractivista, a nivel provincial

\begin{tabular}{|c|c|c|c|c|c|c|c|c|}
\hline Provincia & $\begin{array}{l}\text { Esperanza } \\
\text { de vida } \\
\text { al nacer } \\
\text { (años)a }\end{array}$ & $\begin{array}{l}\text { Años pro- } \\
\text { medio de } \\
\text { escolaridad } \\
\text { (años)b }\end{array}$ & $\begin{array}{l}\text { Años espe- } \\
\text { rados de } \\
\text { escolaridad } \\
\text { (años)c }\end{array}$ & $\begin{array}{c}\text { VAB per } \\
\text { cápita } \\
(\$) d\end{array}$ & $\begin{array}{l}\text { Índice de } \\
\text { esperanza } \\
\text { de vidae }\end{array}$ & $\begin{array}{c}\text { Índice de } \\
\text { educa- } \\
\text { cióne }\end{array}$ & $\begin{array}{l}\text { Índice } \\
\text { VABe }\end{array}$ & $\begin{array}{c}\text { Índice de } \\
\text { Desarrollo } \\
\text { Humano } \\
\text { (IDH)e }\end{array}$ \\
\hline Azuay & 78,6 & 10 & 14,8 & 4717,6 & 0,902 & 0,745 & 0,582 & 0,731 \\
\hline Bolívar & 76,4 & 9,1 & 14,5 & 2408,8 & 0,868 & 0,707 & 0,481 & 0,665 \\
\hline Cañar & 77,3 & 8,5 & 13,3 & 3135,1 & 0,882 & 0,653 & 0,520 & 0,669 \\
\hline Carchi & 77 & 9,2 & 14,3 & 2403,8 & 0,877 & 0,704 & 0,480 & 0,667 \\
\hline Chimborazo & 75,6 & 8,7 & 14,3 & 4491,6 & 0,855 & 0,687 & 0,575 & 0,696 \\
\hline Cotopaxi & 76,1 & 8,9 & 14,1 & 2404,3 & 0,863 & 0,689 & 0,480 & 0,659 \\
\hline El Oro & 76,2 & 10,3 & 14,0 & 4793,6 & 0,865 & 0,733 & 0,585 & 0,718 \\
\hline Esmeraldas & 74,3 & 10 & 13,8 & 2520,7 & 0,835 & 0,717 & 0,487 & 0,663 \\
\hline Galápagos & 77 & 12,4 & 13,5 & 6566,0 & 0,877 & 0,787 & 0,632 & 0,758 \\
\hline Guayas & 75 & 10,7 & 13,9 & 6426,7 & 0,846 & 0,742 & 0,629 & 0,734 \\
\hline Imbabura & 77 & 9,7 & 14,6 & 3697,5 & 0,877 & 0,729 & 0,545 & 0,704 \\
\hline Loja & 78,7 & 10,5 & 14,2 & 4292,9 & 0,903 & 0,745 & 0,568 & 0,726 \\
\hline Los Ríos & 73,6 & 9 & 13,5 & 3600,1 & 0,825 & 0,674 & 0,541 & 0,670 \\
\hline Manabí & 75,8 & 9,3 & 14,1 & 4093,2 & 0,858 & 0,700 & 0,561 & 0,696 \\
\hline Morona Santiago & 75,4 & 10,3 & 13,8 & 2277,5 & 0,852 & 0,727 & 0,472 & 0,664 \\
\hline Nacional & 76,7 & 10,2 & 14,2 & 5160,9 & 0,872 & 0,734 & 0,596 & 0,725 \\
\hline Napo & 75,6 & 10 & 13,6 & 3053,5 & 0,855 & 0,711 & 0,516 & 0,680 \\
\hline Orellana & 75,6 & 9,5 & 13,8 & 2714,3 & 0,855 & 0,700 & 0,499 & 0,668 \\
\hline Pastaza & 75,6 & 10,3 & 14,3 & 2369,3 & 0,855 & 0,741 & 0,478 & 0,672 \\
\hline Pichincha & 77,8 & 11,6 & 15,1 & 9599,8 & 0,889 & 0,807 & 0,689 & 0,791 \\
\hline Santa Elena & 77,9 & 9,5 & 13,7 & 1949,0 & 0,891 & 0,697 & 0,449 & 0,653 \\
\hline Santo Domingo & 75,6 & 9,9 & 13,5 & 3961,8 & 0,855 & 0,704 & 0,556 & 0,694 \\
\hline Sucumbíos & 75,6 & 10 & 13,9 & 3466,7 & 0,855 & 0,720 & 0,536 & 0,691 \\
\hline Tungurahua & 77,2 & 10 & 14,9 & 4124,8 & 0,880 & 0,747 & 0,562 & 0,718 \\
\hline Zamora Chinchipe & 75,6 & 9,9 & 13,5 & 2971,9 & 0,855 & 0,704 & 0,512 & 0,676 \\
\hline
\end{tabular}

Fuente: a) Compendio Estadístico del año 2016 del INEC, b) ENEMDU 2017 (INEC) en SICES, c) Variable construida a partir de la sintaxis proporcionada SICES, utilizando los datos de ENEMDU de diciembre de 2017 d) Cuentas Regionales del BCE 2016 (sustraída la cuenta de "Explotación de minas y canteras"), población de la ENEMDU de diciembre 2017, e) cálculos propios 
Como se puede observar en la tabla 4, a pesar de que el IDH está construido de tal forma que se penalice cuando los progresos alcanzados en las dimensiones sean desiguales, se puede notar una clara distorsión del IDH para ciertas provincias.

Como se puede apreciar, a nivel nacional la afectación de utilizar el VAB no extractivista para construir el IDH es mínima, pues el IDH solo disminuye de 0,728 a 0,725 . No obstante, a nivel provincial se crea una gran distorsión. Una vez restado el componente extractivista del VAB, el IDH de todas las provincias petroleras se reduce de manera importante. En la tabla 5 se muestra que mientras mayor es la disminución en el componente del VAB, mayor es la caída en el IDH respectivo. Este hecho es también visible en el gráfico 2, donde se muestra que las provincias que se dedican a actividades extractivistas sufren pérdidas en el IDH, en función de la disminución de su VAB. 
Tabla 5: Comparación IDH e IDH no extractivista

$\left.\begin{array}{|r|l|c|c|c|}\hline & \multicolumn{1}{|c|}{\text { Provincia }} & \text { IDH no extractivista } & \text { Pérdida IDH } & \text { Pérdida posición } \\ \hline 1 & \text { Pichincha } & 0,791 & - & 0 \\ \hline 2 & \text { Galápagos } & 0,758 & - & 1 \\ \hline 3 & \text { Guayas } & 0,734 & - & 2 \\ \hline 4 & \text { Azuay } & 0,731 & - & 2 \\ \hline 5 & \text { Loja } & 0,726 & - & 3 \\ \hline 6 & \text { Nacional } & 0,725 & -0,003 & 1 \\ \hline 7 & \text { El Oro } & 0,718 & - & 2 \\ \hline 8 & \text { Tungurahua } & 0,718 & - & 2 \\ \hline 9 & \text { Imbabura } & 0,704 & - & 3 \\ \hline 10 & \text { Chimborazo } & 0,696 & - & 3 \\ \hline 11 & \text { Manabí } & 0,696 & - & 3 \\ \hline 12 & \text { Santo Domingo } & 0,694 & - & 3 \\ \hline 13 & \text { Sucumbíos } & 0,691 & -0,055 & -9 \\ \hline 14 & \text { Napo } & 0,680 & - & 3 \\ \hline 15 & \text { Zamora Chinchipe } & 0,676 & - & 3 \\ \hline 16 & \text { Pastaza } & 0,672 & -0,033 & -5 \\ \hline 17 & \text { Los Ríos } & 0,670 & - & 2 \\ \hline 18 & \text { Cañar } & 0,669 & - & 2 \\ \hline 19 & \text { Orellana } & 0,668 & -0,107 & -17 \\ \hline 20 & \text { Carchi } & 0,667 & - & 2 \\ \hline 21 & \text { Bolívar } & 0,665 & - & 2 \\ \hline 22 & \text { Morona Santiago } & 0,664 & - & 2 \\ \hline 23 & \text { Esmeraldas } & 0,663 & -0,017 & -7 \\ \hline 24 & \text { Cotopaxi } & 0,659 & - & 1 \\ \hline 25 & \text { Santa Elena } & 0,653 & -0,014 & -4 \\ \hline\end{array}\right\}$ Desarrollo Humano Alto Humano Medio

Fuente: cálculos propios

Además, como se puede notar, sufrieron grandes disminuciones en su ahora son menos las provincias que cuen- nivel de desarrollo humano, pasando totan con un desarrollo humano alto y más das a ser provincias con desarrollo humalas provincias que muestran un desarrollo no medio en lugar de alto, siendo Orellahumano medio. Las provincias petroleras na la más afectada. Asimismo, se puede 
observar que perdieron posiciones en el diecisiete escalones. Esto demuestra que ranking, por ejemplo, Orellana pasó de el IDH se distorsiona debido a las altas ciser la segunda provincia con mayor de- fras en uno de sus componentes, siendo sarrollo humano de Ecuador a ocupar el en este caso el VAB per cápita el causante puesto diecinueve en la lista, perdiendo de la distorsión.

\section{Gráfico 2: Comparación IDH e IDH no extractivista}

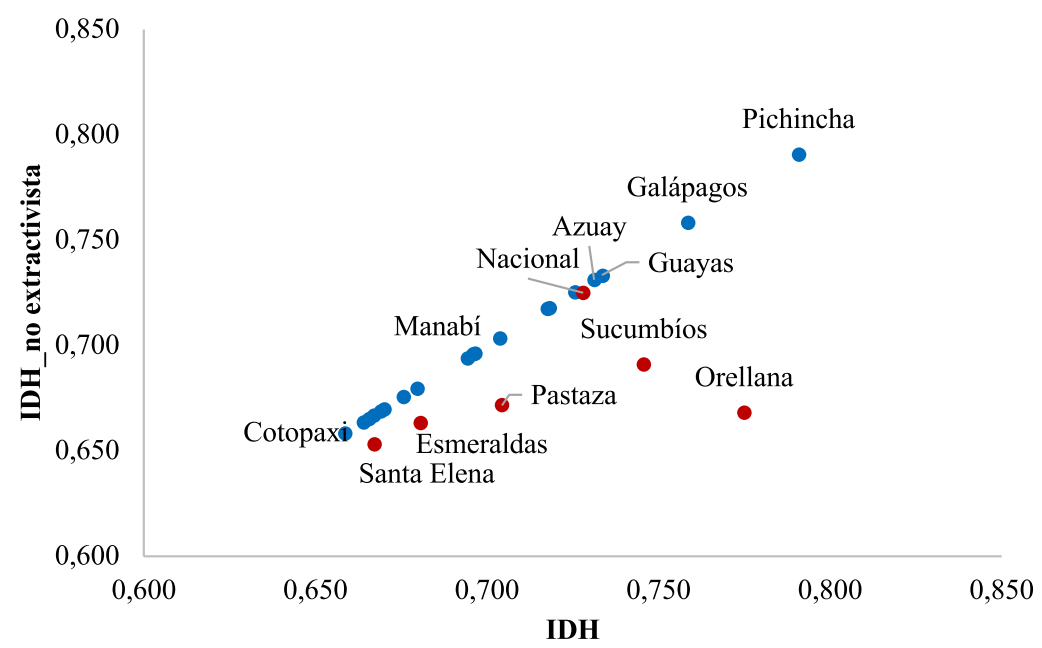

Fuente: cálculos propios 


\section{DISCUSIÓN}

A pesar de que la distorsión ocasionada por el VAB per cápita se podría aliviar modificando el límite máximo del índice de estándar de vida, se considera que la alternativa más adecuada, es utilizar el VAB per cápita no extractivista dado que los ingresos generados por la explotación de minas y canteras o explotación petrolera no se reinvierten en su totalidad en los cantones o provincias donde ocurren las actividades extractivistas.

Según la legislación ecuatoriana, todos los Gobiernos Autónomos Descentralizados (GADs) tienen el $10 \%$ de participación de los ingresos no permanentes del Presupuesto General del Estado, lo que incluye las rentas generadas por el sector hidrocarburífero privado; mientras que la totalidad del excedente generado por el sector público de hidrocarburos es dirigido al Gobierno Central. Adicional a ello, en los GADs en donde se realizan las actividades extractivistas, se destina el $12 \%$ de las utilidades de los trabajadores de empresas mineras y petroleras a proyectos de desarrollo, los cuales otorgan prioridad a las provincias y cantones cercanos a las zonas de explotación (Iturralde \& Jarrín, 2015). No obstante, se debate si en efecto estas políticas logran una redistribución de las rentas extractivas y cuál es su impacto en la calidad de la vida de las personas, así como cuáles son los montos que efectivamente se destinan a las áreas donde ocurre la explotación (Iturralde \& Jarrín, 2015). Por otro lado, se argumenta que estas inversiones responden a intereses políticos, y que el extractivismo en realidad no puede contribuir a un desarrollo humano sostenible (Latorre, 2015).

En este sentido para evitar la distorsión causada por las actividades extractivistas en la dimensión de estándar de vida, resulta pertinente sustraer este componente del VAB y así utilizar el IDH no extractivista, en lugar del IDH convencional, para el caso ecuatoriano. Cabe mencionar también, que si bien existe un índice de desarrollo humano ajustado por desigualdad (IDH-D), como se mencionó al inicio, resulta pertinente primero ajustar el IDH en función del componente extractivista antes de hacerlo por el nivel de desigualdad. Pues el IDH-D, se construye en función del IDH, con las mismas dimensiones y variables, con la diferencia de que antes de agregar las dimensiones, se ajusta cada una de ellas mediante un coeficiente de desigualdad. Sin embargo, dado que la dimensión de estándar de vida se ajusta por el nivel de desigualdad en los ingresos disponibles 
de las familias o en el consumo per cápita (PNUD, 2016b), todavía se estaría ignorando el hecho de que solo se reinvierte un porcentaje de los ingresos generados por las actividades extractivistas y que el
$\mathrm{IDH}$, particularmente, de las provincias donde se realizan actividades extractivistas se ve distorsionado por los altos ingresos registrados debido a estas actividades.

\section{CONCLUSIÓN}

El IDH revolucionó -hace ya casi tres décadas - la forma en la que se cuantificaba el desarrollo de los países. Este enfoque logró que se analice el desarrollo de los países no solo en función de los ingresos sino en función de la calidad de vida de sus habitantes, al incorporar las dimensiones de salud y educación, además del nivel de ingresos.

Si bien este índice ha servido para estos fines y sigue siendo uno de los referentes a nivel mundial para comparar a los países según su nivel de desarrollo, no es suficientemente informativo sobre la realidad de cada país. Dado que este índice es calculado a nivel internacional depende de la información que se encuentre disponible para la mayoría de países por lo que ignora aspectos relevantes sobre el contexto de cada país.

Si se calcula el IDH para Ecuador a nivel nacional, este esconde las disparidades existentes entre las provincias. Pues, según el IDH, Ecuador tiene un desarrollo humano alto, sin embargo, cuando se observa el desarrollo de las provin- cias, se encuentra que en realidad trece de las veinticuatro provincias presentan un desarrollo humano medio, cifra que se agrava cuando se calcula el IDH no extractivista.

Si bien, construir indicadores a nivel provincial alivia este problema ya que permite visibilizar de una manera más acertada cómo se encuentra el desarrollo humano en las distintas regiones del país, no es suficiente dado que dentro de las mismas provincias existe un nivel de desigualdad muy elevado por lo que se debería construir los indicadores al nivel más desagregado posible.

Adicionalmente, se observó que el IDH se distorsiona por altas cifras en uno de sus componentes por lo que requiere de ajustes para que refleje de mejor manera el desarrollo humano a nivel provincial en Ecuador. En este sentido, se debe excluir el componente de explotación de minas y canteras del VAB per cápita, caso contrario, el IDH de las provincias que realizan actividades extractivistas se ve distorsionado. 
Las implicaciones de que un ín- líticas, organismos no gubernamentales dice no refleje la realidad del país que y otras organizaciones que trabajan conse está evaluando no son menores. Esto podría causar que se direccionen incotinuamente en programas de desarrollo. rrectamente políticas y proyectos, y que a su vez se desvíen esfuerzos y recursos valiosos que tenían por objetivo contribuir al desarrollo de un país o región. Por ello, es necesario revisar e innovar las estadísticas, así como construirlas a niveles más desagregados para poder informar de mejor manera a los hacedores de po-

Este esfuerzo de construir el IDH a nivel provincial y corregirlo para que realice una medición más adecuada del desarrollo a nivel provincial para Ecuador es un aporte relevante, sin embargo, sería importante también añadir otros indicadores, por ejemplo, sobre la calidad de la educación u otros aspectos que sean relevantes para el contexto del país. 


\section{REFERENCIAS BIBLIOGRÁFICAS}

Biswas-Diener, R., Diener, E., \& Tamir, M. (2004). The Psychology of Subjective Well-Being. Obtenido de Daedalus, Vol. 133, No. 2, On Happiness, pp. 18-25. MIT Press: http:// www.jstor.org/stable/20027909

Burgos, S., \& Cando, F. (2016). Pobreza multidimensional: índice de Alkire y Foster para Ecuador. Economía, XLI, 42, http://www.saber.ula. ve/handle/123456789/43179

Iturralde, P., \& Jarrín, S. (2015). En Latorre, S. (Coordinadora), Renta extractiva y territorialización de la política pública. Extractivismo al debate: Aportes para los Gobiernos Autónomos Descentralizados. Quito: Ediciones Abya-Yala.

Kroll, C. (2015). Global Development and Happiness: How Can Data on Subjective Well-Being Inform Development Theory and Practice? Oxford Development Studies, 43:3, 281-309, DOI: 10.1080/13600818.2015.1067293

Latorre, S. (2015). Latorre, S. (Coordinadora), Visibilidades e invisibilidades del extractivismo en Ecuador: insumos para el debate. Extractivismo al debate: Aportes para los Gobiernos Autónomos Descentralizados (págs. 15-56). Quito: Ediciones Abya-Yala.
Nussbaum, M. (2003). Capabilities as Fundamental Entilements: Sen and Social Justice. Obtenido de Feminist Economics, 9:2-3, 33-59.

PNUD. (1990). Desarrollo Humano Informe. Bogotá: Tercer Mundo Editores.

PNUD. (2003). El conflicto, callejón con salida: Informe Nacional de Desarrollo Humano para Colombia. Bogotá: PNUD.

PNUD. (2006). Venciendo el temor:(In) Seguridad ciudadana y desarroIlo humano Costa Rica. Informe de Desarrollo Humano 2015. San José: UNDP.

PNUD. (2009). Assessment of Development Results: Evaluation of UNDP Constribution - Argentina. Argentina: PNUD.

PNUD. (2010). Informe sobre Desarrollo Humano: Edición del Vigésimo Aniversario. Nueva York: Communications Development Incorporated.

PNUD. (2015). Training Material for Producing National Human Development Reports (Occasional Paper). New York: PNUD.

PNUD. (2016a). Informe sobre Desarrollo Humano: Desarrollo humano para todas las personas. Nueva York: Communications Development Incorporated. 
PNUD. (2016b). Nota Técnica. Informe de Desarrollo Humano: Desarrollo Humano para todos.

Ranis, G., Stewart, F. \& Samman, E. (2006). Human Development: Beyond the Human Development Index. Journal of Human Development, 7:3, 323-358, DOl: 10.1080/14649880600815917.

Sachs, W. (2015). Planet Dialectics: Explaration in Environment and Development. Londrés: Zed Books.

Sagar, A., \& Najam, A. (1999). Shaping human development: which way next? Obtenido de Third World
Quarterly, Vol. 20, No. 4 (Aug., 1999), pp. 743-751: http://www. jstor.org/stable/3993586

Sen, A. (1999). Development as a freedom. Oxford: Oxford University Press.

Sen, A. (2012). Develpment as Capability Expansion. En J. Defilippis, \& S. Saegert, The Commutiy Development Reader (págs. 319 - 327). New York y Londres: Routledge: Taylor and Francis Group.

Todaro, M., \& Smith, S. (2015). Economic Development. Doceava Edición. Pearson. 